\title{
Experience with the Silicon Strip Detector of ALICE
}

\section{G.J.L. Nooren* for the ALICE collaboration}

Institute for Subatomic Physics Utrecht University P.O.B. 80000, 3508 TA Utrecht, the

Netherlands

E-mail: noerendaikhef,nd

The Silicon Strip Detector (SSD) forms the two outermost layers of the ALICE Inner Tracking System (ITS), connecting the TPC with the inner layers of the ITS. The SSD consists of 1698 double-sided silicon microstrip modules, $95 \mu \mathrm{m}$ pitch, distributed in two cylindrical barrels, whose radii are $38 \mathrm{~cm}$ and $43 \mathrm{~cm}$, respectively. With 4.8 square meters it is today's largest installed double-sided strip detector. The design and construction will be discussed, both in view of the requirements of a decade ago and in the light of today's achieved performance. Like the other LHC detectors, the SSD is waiting for beam after extensive commissioning with cosmics. These commissioning results will be presented. Cosmics data without magnetic field were used for alignment and for relative gain calibration. Together with the measured noise this allowed the signal-to-noise ratio to be determined as well as the charge matching quality. Cosmics data collected with magnetic field were used for energy-loss calibration.

9th International Conference on Large Scale Applications and Radiation Hardness of Semiconductor Detectors, RD09

September 30-October 2, 2009

Florence, Italy

\footnotetext{
* Speaker.
} 


\section{General layout of the detector}

The Silicon Strip Detector (SSD) forms the two outermost of the six layers of the Inner Tracking System (ITS) of ALICE[U, 口, [] at the LHC. It connects the ITS to the main tracker, the TPC, whilst providing additional particle identification, especially for low momentum $(<100 \mathrm{MeV} / c)$ particles. The SSD consists of 1698 double-sided silicon microstrip modules, mounted on 72 ladders distributed over two concentric cylindrical barrels, whose radii are $38 \mathrm{~cm}$ and $43 \mathrm{~cm}$, respectively. It covers the full azimuth and rapidities $|\eta|<0.9$ for all vertices located within the length of the interaction diamond $( \pm 1 \sigma)$, i.e. $10.6 \mathrm{~cm}$ along the beam direction. With 4.8 square meters this is today's largest installed double-sided strip detector. The strips have $95 \mu \mathrm{m}$ pitch and are oriented on the $\mathrm{P}(\mathrm{N})$-side of the sensor at an angle of 7.5(27.5) mrad with respect to the beam (z) direction. For layer 5(6), the $\mathrm{P}(\mathrm{N})$-side is facing the beamline. This orientation together with the small stereo angle reduce the fake track probability significantly. The near parallelism to the magnetic field ensures that the best position resolution is in the bending direction.

Double-sided micro-strips have been selected rather than single-sided ones because they introduce less material in the active volume. In addition, they allow to correlate the pulse heights from the two sides, to help resolve ambiguities [团]. A detection module consists of one sensor connected to two hybrids, one for each sensor side, both mounted on the top side. Each hybrid carries six front-end chips (HAL25)[[]], on the side facing the sensor. Flexible aluminium microcables, bonded directly to sensor and chips, make this three dimensional layout possible.

The HAL25 contains 128 identical channels. It amplifies, shapes and stores analog signals from the sensor and provides an interface to the readout system. The pre-amplifier's integrator limits the maximum strip hit rate to $\approx 50 \mathrm{~Hz}$. The analog signals are stored in a sample-hold circuit, controlled by an external HOLD signal derived from the level-0 trigger (L0). The analog signals are read out serially at a maximum speed of 10 Msamples/s, which limits the L0 rate to $5 \mathrm{kHz}$. The linear range corresponds to $\approx 13 \mathrm{MIP}$.

Electronics located at each end of a ladder [ 6$]$ contains two types of ASICs. The ALCAPONE distributes the power to the HAL25, protects them from latch-up and controls their settings through the JTAG interface. The ALABUF couples the signals from the front-ends, which are at sensor bias voltage, to the readout system which is at common ground, via capacitors. The three types of ASICs: HAL25, ALCAPONE and ALABUF, are made in radiation tolerant $0.25 \mu \mathrm{m}$ technology. Electrically each half ladder is independent from the other half, but they share the mechanics and cooling.

The Front-End ReadOut Modules (FEROM)[D] are located in eight crates outside the ALICE L3 solenoid. Their distance to the EndCap electronics is $30 \mathrm{~m}$. This system controls the readout of the front-end chips and digitises the analog data from all modules in parallel upon receipt of an L1 trigger. The data are corrected for pedestal and common mode on the fly. The zero-suppressed data are stored in a multiple-event buffer, allowing a new L0-L1-L2 cycle to start before all data are transferred to the DAQ system. Each FEROM is connected to the DAQ system through two optical links allowing a read-out rate of 300 non-suppressed events per second. Because of its distance to the beamline this system is built in conventional technology, apart from the crate power supply which is radiation tolerant. The completed SSD in its assembly position is shown in fig. $\mathbb{\text { m. }}$ 


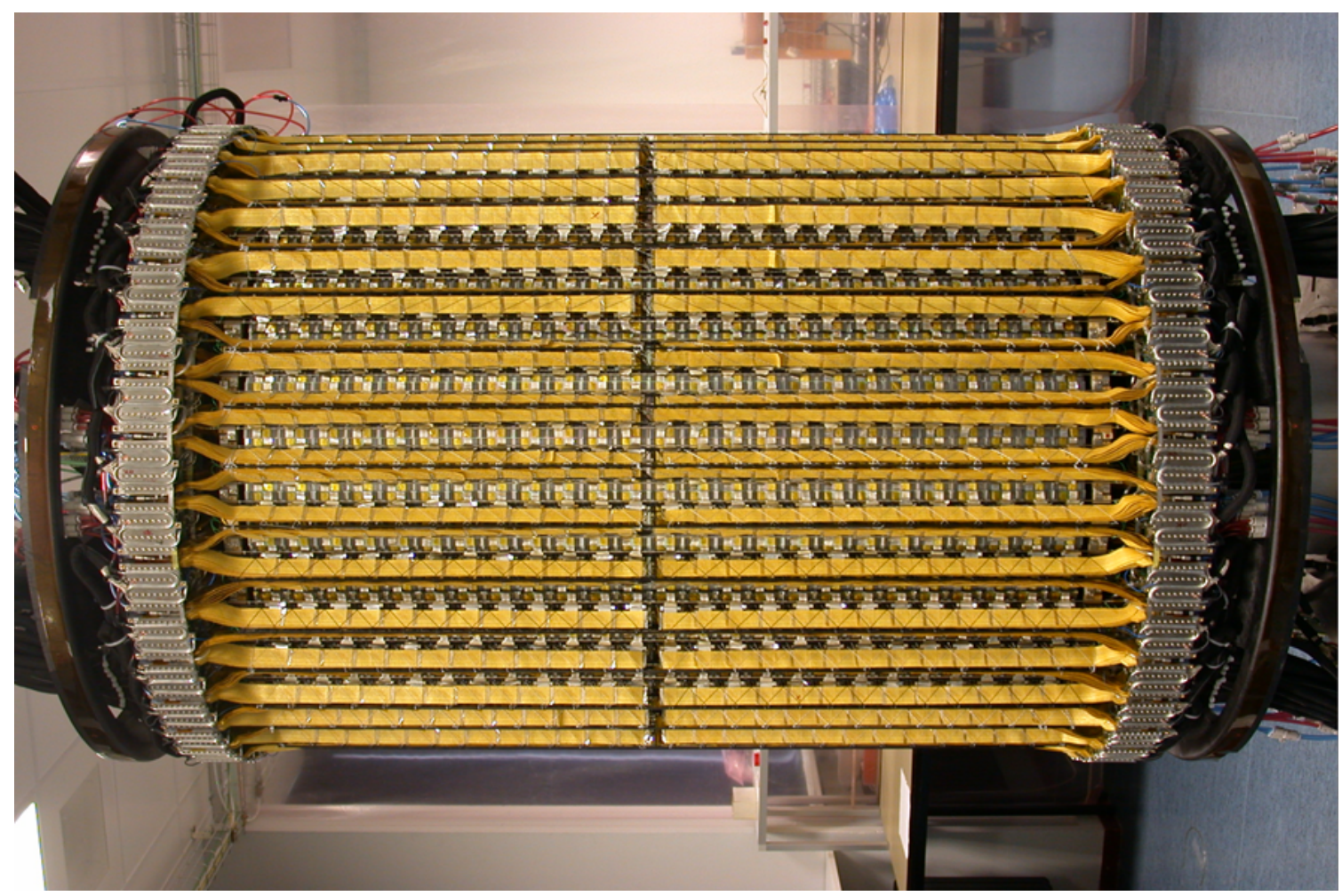

Figure 1: The completed Silicon Strip Detector. The ladders of the outer layer are clearly recognizeable by the laddercables on both sides of each carbon-fibre frame. These cables (gold colour) connect the hybrids to the watercooled EndCaps which are visible at each ladder end.

\section{Design principles}

The construction of the SSD is described elsewhere[四] in more detail. We focus here on the underlying design principles that are special for this detector. Throughout the design phase special care was taken of:

- mechanical stability, knowing that survey after installation would not be possible;

- low material budget, to minimize the scattering of the particles on their way to the main tracking device TPC;

- grounding and power distribution, as in the conceptual design phase the decision was made to digitize the signals only outside the magnet, thus involving long cable lengths.

\subsection{Mechanics}

In order to preserve the position of the sensor relative to the external alignment points, the mechanics was required to be stress-free. This implies that the position of any object shall not be over-constrained, i.e. each degree of freedom is fixed only once, as is shown in the following cases. If we consider the full ITS as a solid body, it has only six degrees of freedom, which are constrained in the following way: one support point suffices to define its position, the second point defines only 


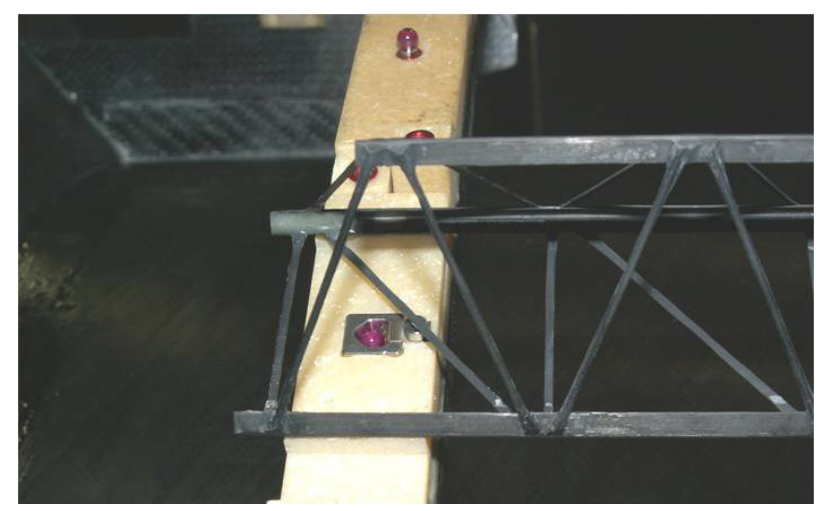

Figure 2: Ladder without modules on one of its supports. The carbonfibre frame with its G10 mounting block rests on its support ring. On top the support for the neighbouring ladder is seen, made of precisely machined G10 and a ruby sphere. This ruby together with the V-shaped hole in the block on the ladder defines the two in-plane coordinates. The other coordinate and one rotational degree of freedom are fixed by the mounting block.

two rotations and the third point defines the remaining rotation angle. The same principle is used for the positioning of the ladders: one support defines the three coordinates and one angle, the other support the remaining angles. Figure $\square$ shows the end of a ladder (without modules!) on its support. The silicon sensor is mounted onto a carbon-fibre frame via three support pins glued to its surface. The flexibility of the pins and the glue accomodate any stress due to thermal expansion differences. The centre-of-gravity of these pins coincides with the centre-of-gravity of the silicon, so this will remain at the same position.

\subsection{Mass and radiation length}

All design decisions were driven by the need to keep the radiation thickness as small as possible. The functionalitiy of the modules was kept minimal, with functions like power control and signal treatment deferred to the end of the ladders, which are outside the detection volume. Extensive use is made of aluminium-on-polyimide flatcables [8] $]$. Apart from its low radiation thickness, aluminium allows direct bonding to the chips and to other cables. The hybrids are made of a flex of two layers of this material for the electrical connections, while the stiffness and thermal conductivity are provided by a carbon fibre stiffener. Like with the carbon fibre ladderframes, the fibres are mainly oriented in the "useful" direction, in this case the direction of the heat flow. This required manual fabrication techniques, but leads to large mass saving compared to the use of woven raw material. The HAL25 front-end chips are thinned to $150 \mu \mathrm{m}$. The resulting mass and radiation thickness are $6.3 \mathrm{~g}$ and $0.85 \% X_{0}$ for an average module, i.e. all structures on the ladder within the acceptance are equally attributed to each module and normal incidence is assumed. The radiation thickness of the inner and outer shields of the SSD is $0.53 \% X_{0}$ and $0.02 \% X_{0}$, respectively. The contribution of the major components is shown in fig. [ ] for a ladder (left) and a module (right).

\subsection{Grounding and power distribution}

With double-sided sensors the choice of the ground level is not obvious. For the SSD full symmetry between $\mathrm{P}$ - and $\mathrm{N}$-sides was chosen. This implies not only floating low voltage power 

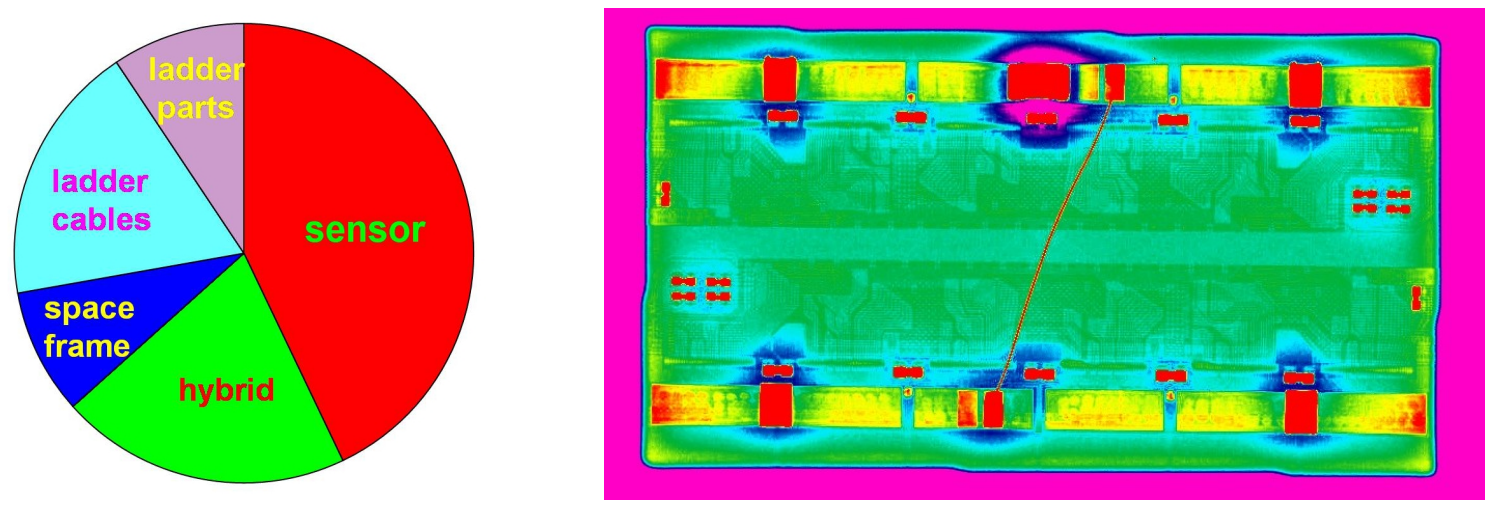

Figure 3: Radiation thickness of the SSD. Left: Contributions of various parts of a ladder. The main contribution to the item "ladder parts" consists of the cooling tubes with their water. Right: X-ray image of a module without ladder in false colour (rainbow: red highest, magenta lowest). Note the scattering around the massive components on the hybrids: four aluminum cooling blocks and capacitors. Beam direction is vertical.

supplies for the charge-sensitive amplifiers and related electronics, but also double bias supplies, each for one side with their interconnection at ground level. The rationale behind this decision is to have the impedances equal for both sides to make the system less sensitive to common mode signals. Two other measures to minimize the influence of external noise are the following. The grounds of the P-and N-hybrids are interconnected directly at the module level, making the charge signal loop minimal. The price to pay here is a rather large capacitor in the sensor area (centre object on top in fig. (B] right). The other measure is the use of differential low-voltage for the transport of the signals both along the ladder and for the long distance to the electronics racks. Even with these measures grounding remains very important. A two level grounding and shielding system was made: a signal ground of the analogue signal and a machine ground to which structures like cabinets, cable trays are connected. These objects are inevitably connected to safety ground in an uncontrolled way. The "official" connection to safety ground is done at the racks at each side of ALICE.

\subsubsection{Principle}

The signal ground and the machine ground are connected to the inner and the outer shields, respectively. The shields are interconnected only at the electronics cabinet. However, there will be a large capacitive coupling due to the proximity and long length of the conductors. Wherever possible, the conductors are laid out in such a way as to minimize the inductance: e.g. several parallel wires instead of one with the same crosssection, interconnect spare pins in connectors, etc. An important question had to be answered: what to do with the ladder structure, made of conductive carbon fibres? The read-out is connected to different sides of ALICE, whereas the ladders mechanically form one piece. As the sensors and the laddercables are close to the ladder structure there is a large capacitive coupling, eventually between the two sides of ALICE. It was decided to reduce the effect of this coupling by connecting both analog grounds to the ladder structure. The low impedance of the ladder structure (few ohms DC) prevents large voltages along the ladder in case of ground loop currents. To reduce the ground currents in this structure, the outer 
shields were interconnected via the ITS structure, which forms a parallel low impedance path. The SSD ladders with their sensors are enclosed in this structure, which acts as a Faraday cage.

\subsubsection{Segmentation}

Ladders and halfladders are grouped in 4 groups of 18. Each group has a mixture of layer 5 and layer 6 ladders such as to accommodate 34 layer 5 and 38 layer 6 ladders. All cables of one group follow the same trajectory and are kept as tightly together as possible in order to minimise the enclosed areas. The patchpanels have the same segmentation. The analogue and digital signal cables end in one VME crate where their inner shields are connected to signal ground. The power cables (LV and bias) are a bit more distributed due to different segmentation of the power supply units, but remain in the same cabinet. At the ladder ends, the signal grounds of one group are mechanically interconnected, as well as their shields.

\subsubsection{Results}

After its installation the detector was tuned: biasvoltage and several timing issues. In calibration runs with pulser trigger the noise level was $\approx 10 \mathrm{mV}$, much higher than the intrinsic noise measured in the laboratory. This was traced down to the influence of the low voltage power supplies, possibly in combination with power distribution cables. Being common to hybrids with the same power supply, the noise could be reduced by correcting for the common mode signal. This correction was implemented in the FEROM and is done on-the-fly for each event and each HAL25 chip. After this correction the noise is sufficiently reduced to enable zero suppression. Although a signal-to-noise ratio of better than twenty (typically 40) is obtained, research is going on with the supplier to reduce the common mode at its source.

\section{Results of commissioning with cosmics}

During several campaigns, both in 2008 and 2009, ALICE collected cosmics both without field, used for alignment purposes and for relative (P-vs. N-side) gain calibration, and with field, for calibration. For the SSD the fraction of useable events depends on the type of trigger. The trigger provided by the pixel detector [Q] had the highest purity, as all particles detected by the pixel detector have traversed the SSD. In the present study 17 million events with magnetic field were analysed. Obviously not all ladders have the same number of hits. Figure 1 shows the distribution of hits in the two layers in cosmics runs without field. The results shown below refer only to the ladders with enough statistics. The calibration and alignment of the remaining ladders can only be done when beam is available.

\subsection{Alignment}

Using the four hits of a straight cosmic track through the SSD one can measure the relative orientation of the two layers by looking at the distance between the line defined by one layer and the actual hits in the other layer. Figure $\square$ shows the distribution of these distances after applying the pre-installation survey data to cosmics data taken without magnetic field. The results clearly indicate that the mechanical design is performing as expected. Taking into account that four hits are 

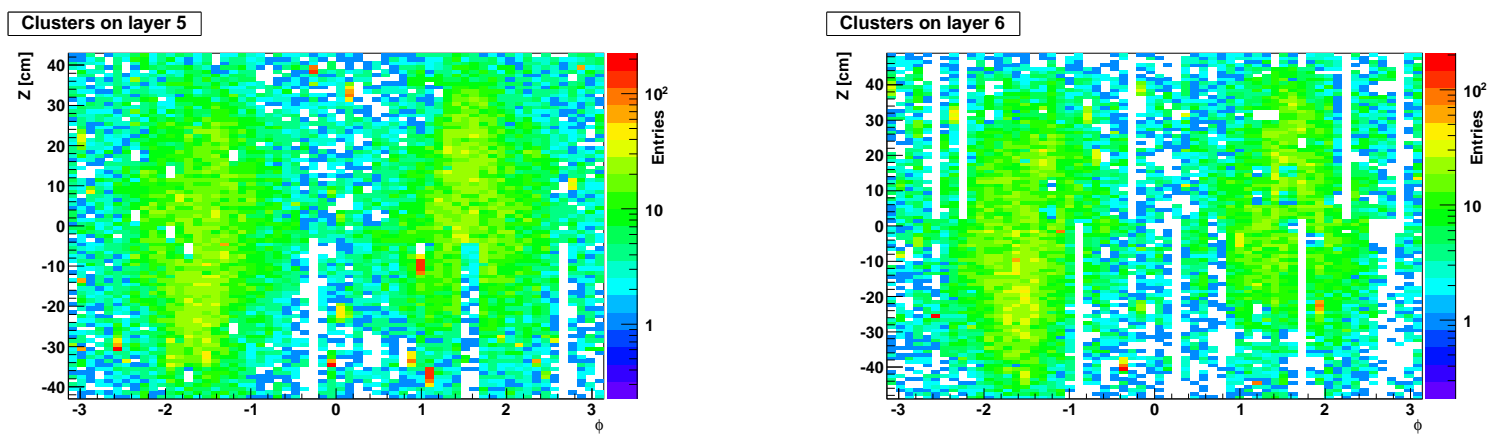

Figure 4: Typical hit pattern of cosmic hits. Vertical axis: $Z$, horizontal axis: $R \phi$. Left: inner layer, right: outer layer. The white areas represent modules or ladders which were either dead or switched off during the datataking.
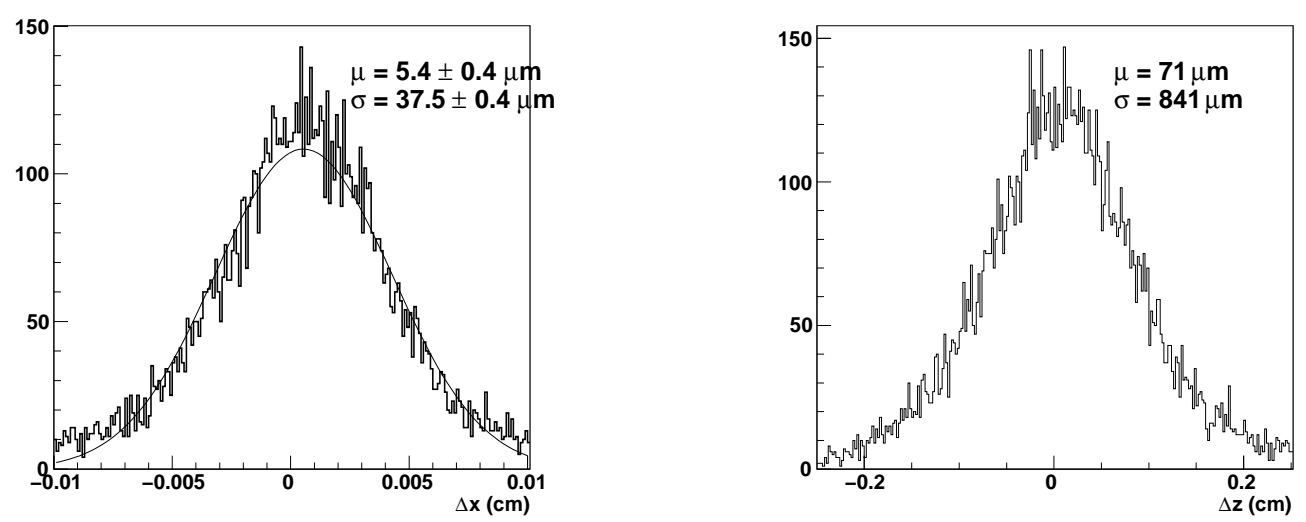

Figure 5: Histogram of the the residuals of $510^{4}$ tracks after fitting the hit position to the two layers. Left: in $R \phi$, right: in $Z$.

involved in the procedure the resulting resolution is $27 \mu \mathrm{m}$, which compares well with the $20 \mu \mathrm{m}$ measured[田] on modules in beam tests .

\subsection{Calibration}

For the relative calibration of $\mathrm{P}$ - and $\mathrm{N}$-side signals all particle tracks can be used, but even then the cosmics data do not contain enough hits to allow calibration of individual modules. As a first step all data of all layers were combined. Charge clusters from one side of a sensor were coupled to clusters on the other side whenever the resulting position was inside the sensor area. Both one- and two-dimensional distributions are shown in fig. $\mathrm{Q}$. The gains for the P- and $\mathrm{N}$-side were adjusted such that the peaks in the cluster charge distributions are at the same position. The absolute calibration is still in progress in combination with the SDD and the TPC. Only P-N pairs with a charge ratio within certain limits (present limits are the red lines in fig. (6) will be used in the subsequent processing. This charge matching reduces hits from noise and ambiguities. After removal of extremely noisy strips ${ }^{1}$ the number of noise hits in a module is $10^{-4}$ per trigger.

\footnotetext{
${ }^{1}$ The fraction of bad strips (dead, pedestal, noise) is less than $2 \%$.
} 

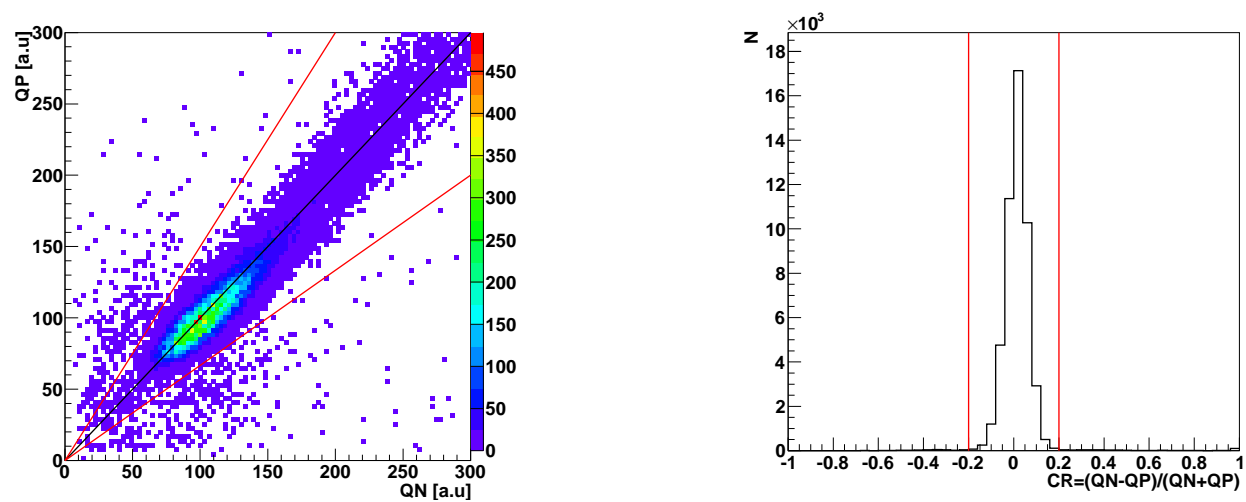

Figure 6: Charge distributions of tracks. Left: histogram of the cluster charge of P-side vs. N-side. Right: the charge ratio defined as the normalised difference of $\mathrm{N}$ - and P-charge. The red lines show the position of the cuts used in the charge matching.

\section{Conclusion}

The tests of the installed detector have practically confirmed the results from previous (beam)tests and have shown the design to be intrinsically correct. Calibration and alignment have reached the point where more cosmics data will hardly improve the situation. Other particles and realistic tracks are needed to reach absolute calibration and detect remaining (small) misalignments. The latter is expected to improve the measured resolution. Although the noise level can still be reduced it is sufficiently low for data taking with beams. This was confirmed by the detection and tracking of particles produced by the beam on 11 September 2008 during early LHC tests.

\section{References}

[1] ALICE Collaboration, Intern. Journal Modern Physics E16 7\&8(2007) 2445-2450.

[2] ALICE Collaboration, J. Phys. G: Nucl. Part. Phys. 30 (2004) 1517-1763

[3] ALICE Collaboration, J. Phys. G: Nucl. Part. Phys. 32 (2006) 1295-2040

[4] O. Sokolov et al., Nucl. Instr. Meth. A562 (2006) 110-119.

[5] C. Hu-Guo et al., Proceedings of the 7th workshop on Electronics for LHC experiments, Stockholm, Sweden, September 2001.

[6] R. Kluit et al, Proceedings of the 7th workshop on Electronics for LHC experiments, Stockholm, Sweden, September 2001.

[7] M.J. Rossewij et al, Proceedings of the 9th workshop on Electronics for LHC experiments, Amsterdam, the Netherlands, September 2003.

[8] M. Bregant et al., Nucl. Instr. Meth. A570 (2007) 312-316.

[9] G. Aglieri Rinella, The ALICE Pixel Trigger system, commissioning and operation, this conference. 